\title{
Implantation of cardiac resynchronization therapy through persistent left superior vena cava
}

\author{
Marcin Michalak ${ }^{1, \mathrm{~A}-\mathrm{F}}$, Szymon Jonik ${ }^{1, \mathrm{~A}-\mathrm{F}}$, Łukasz Januszkiewicz ${ }^{1, \mathrm{~A}-\mathrm{F}}$, Grzegorz Opolski ${ }^{1, \mathrm{~A}-\mathrm{F}}$, Marcin Grabowski $^{1, \mathrm{~A}-\mathrm{F}}$ \\ A - Research concept and design, B - Collection and/or assembly of data, C - Data analysis and interpretation, \\ D - Writing the article, E - Critical revision of the article, F - Final approval of article \\ 1. $1^{\text {st }}$ Department of Cardiology, Medical University of Warsaw
}

Address for correspondence:

Marcin Michalak, $1^{\text {st }}$ Department of Cardiology, Medical University of Warsaw email: mmajkelak@gmail.com

Szymon Jonik, $1^{\text {st }}$ Department of Cardiology, Medical University of Warsaw email: szymonjonik.wum@gmail.com

Łukasz Januszkiewicz, $1^{\text {st }}$ Department of Cardiology, Medical University of Warsaw email: lukasz.jan.januszkiewicz@gmail.com

Grzegorz Opolski, $1^{\text {st }}$ Department of Cardiology, Medical University of Warsaw email: grzegorz.opolski@wum.edu.pl

Marcin Grabowski, $1^{\text {st }}$ Department of Cardiology, Medical University of Warsaw email: marcin.grabowski@wum.edu.pl

Received: 2018-11-21

Revised:

Accepted: 2018-11-26

Final review: 2018-11-26

DOI: $10.24255 / \mathrm{hbj} / 100354$

\section{Key words:}

persistent left superior vena cava, cardiac resynchronization therapy, lead implantation complications

\begin{abstract}
Persistent left superior vena cava (PLSVC) is the most common anomaly of systemic venous return. Its occurrence is found in 0.3 to $0.5 \%$ of the general population and in up to $10 \%$ of patients with congenital heart defects. We present a patient with numerous cardiological burdens who was admitted to our clinic for implantation of cardiac resynchronization therapy with a defibrillator (CRT-D) due to heart failure (NYHA III class) with accompanying permanent atrial fibrillation and left
\end{abstract}

Persistent left superior vena cava (PLSVC) is a rare congenital cardiac anomaly with an incidence of $0.47 \%$ in patients undergoing cardiac electronic device implantation ${ }^{[1]}$, which may hamper this procedure $\mathrm{r}^{[2],[3]}$.

A 66-year-old woman with non-ischemic cardiomyopathy, severe left ventricular dysfunction (ejection fraction, EF 10\%), NYHA class III symptoms, left bundle branch block (LBBB, QRS width $160 \mathrm{~ms}$ ) and permanent atrial fibrillation (AF) was admitted to our clinic for cardiac resynchronization therapy bundle branch block with QRS width of 160 ms. During the implantation in venography we found PLSVC. Despite this anomaly, we found one large lateral branch for introduction of leads and the procedure was successful. PLSVC is usually asymptomatic and found accidentally, as in our patient. Unfortunately, the existence of PLSVC significantly worsens the prognosis of patients undergoing implantation. In case of failure, the only solution is cardiac surgery.

(CRT) with defibrillator implantation. One year before the index hospitalization she underwent minimally invasive mitral valve replacement because of severe symptomatic mitral valve stenosis. Preprocedural EF was $60 \%$. The surgery was uncomplicated except for development of LBBB. During routine 1-month follow-up she was found to be in good general condition without heart failure (HF) symptoms. Three months later she was hospitalized due to HF NYHA class III. The function of the prosthetic valve was normal, but the EF had 
decreased to $40 \%$. After 7 months her EF was rated at $26 \%$, and a month later she was hospitalized due to HF exacerbation to NYHA class IV and her EF had decreased further to $10 \%$. Because of her long history of AF and significantly enlarged left atrium (54 mm), implantation of only a biventricular device without an atrial lead was planned. Vascular access was obtained through the left brachial vein puncture. The atypical position of the wire suggested venous anomaly. Venography revealed PLSVC without connection with the right side confluence (Figure 1A). Using subselective coronary sinus catheters,

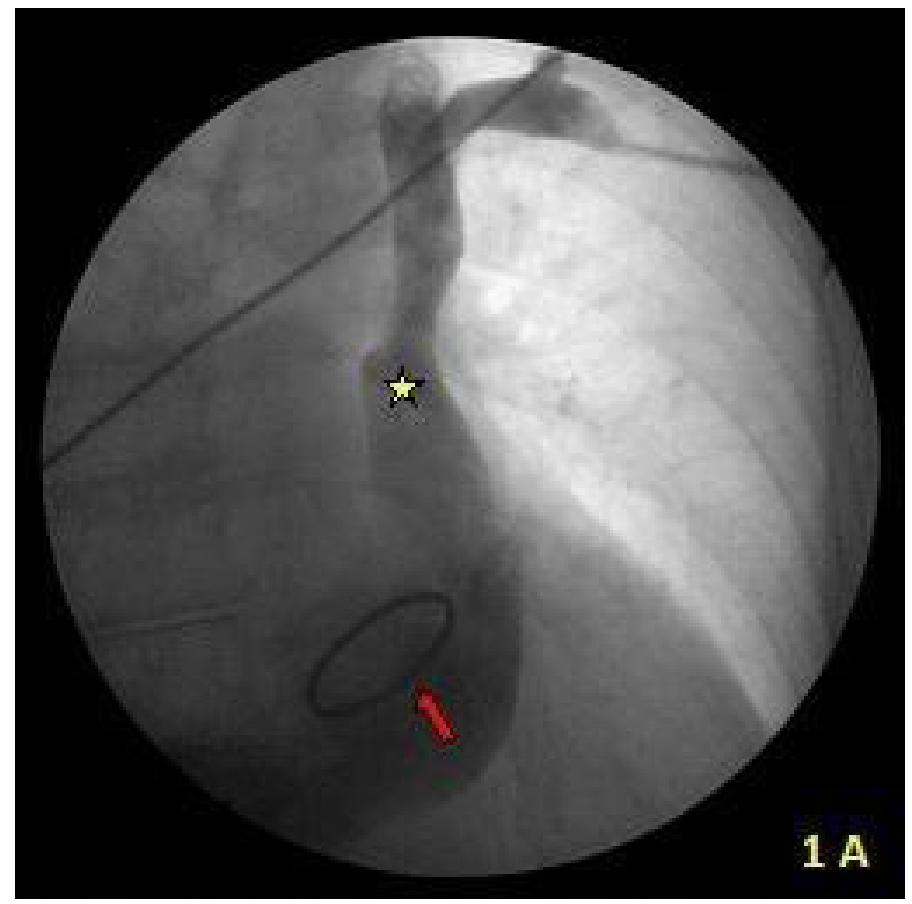

Figure 1A. Left superior vena caval( 3 ) draining into left atrium without connection with right side. Prosthetic mechanical mitral valve( $)$

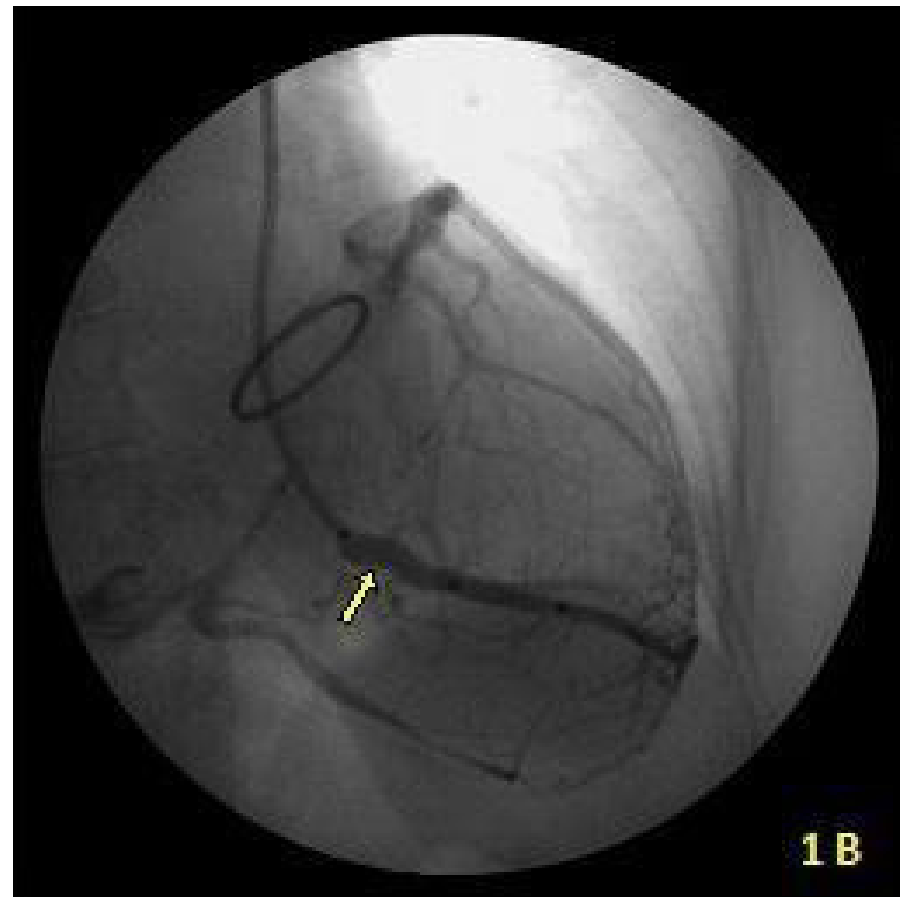

one large lateral branch was found and became the target vein. Selective venography of this branch showed complete anatomy of coronary veins (Figure $1 \mathrm{~B}$ ) and confirmed lack of other potentially achievable lateral branches. The quadripolar left ventricular lead (Biotronik, Sentus OTW 85-QP) was introduced. Pacing with relatively low thresholds (0.7 to 2.0 $\mathrm{V}$ with $0.4 \mathrm{~ms}$ ) was possible from all four pacing rings, but there was an unacceptably low margin between the pacing threshold and phrenic nerve stimulation on the distal two electrodes. Then a single-coil active-fixation defibrillation lead (Biotronik, Protego ProMRI S65) was placed at the apex of the right ventricle. The final position of leads is shown in Figure 1C, D. Preprocedural and postprocedural ECG is shown in
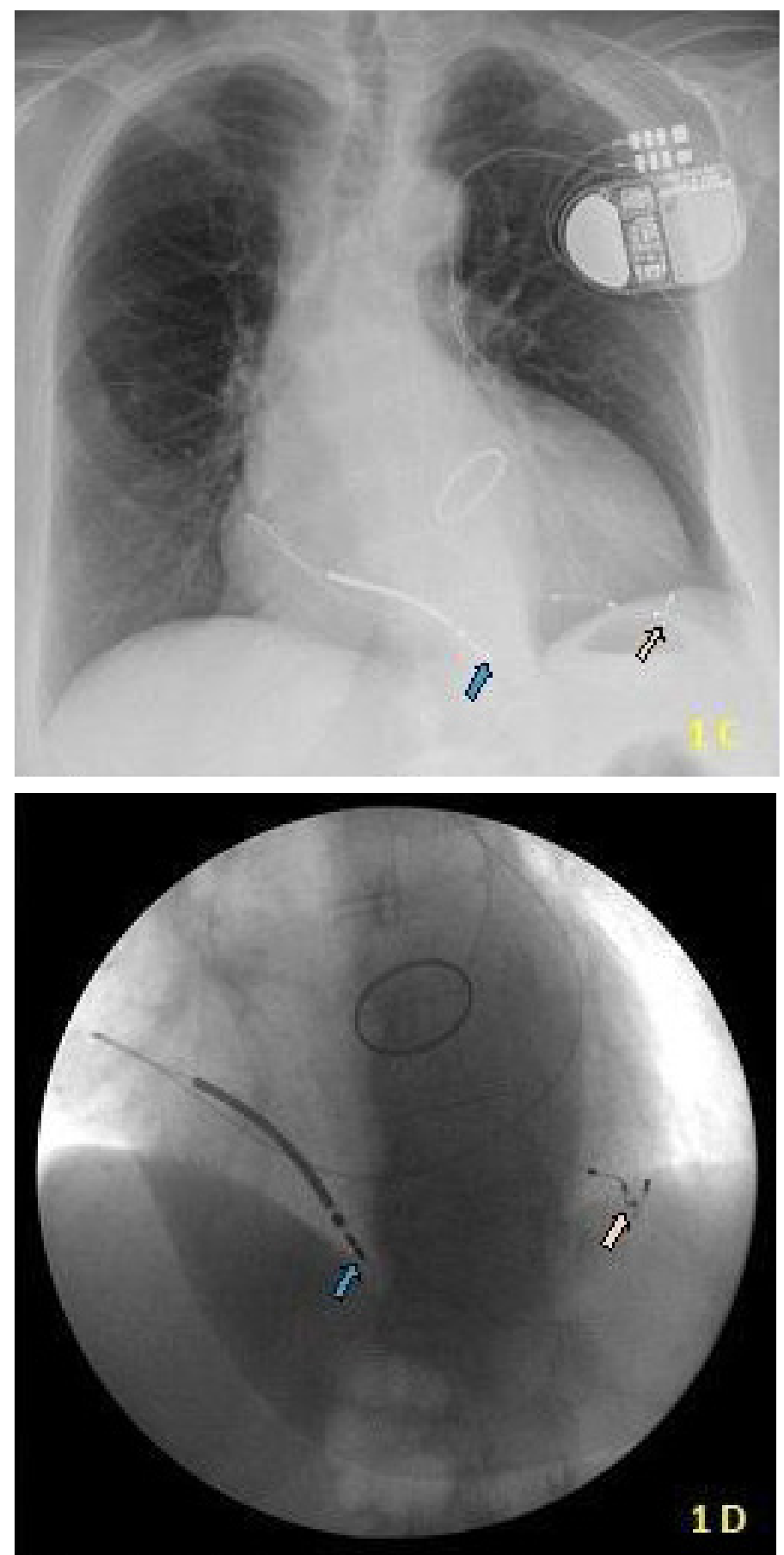

Figure 1C, D. Radiography: Various X-ray projections - anterior-posterior (C) and left anterior oblique (D) showing the correct positions of the right ventricular ( $)$ and the left ventricular lead 
Figure 1E, F. At 3-month follow-up the patient responded to CRT therapy. Her EF had increased to $36 \%$, and end-systolic and end-diastolic left ventricular diameters had decreased to 45 and $55 \mathrm{~mm}$, respectively (before the procedure: 52 and 69
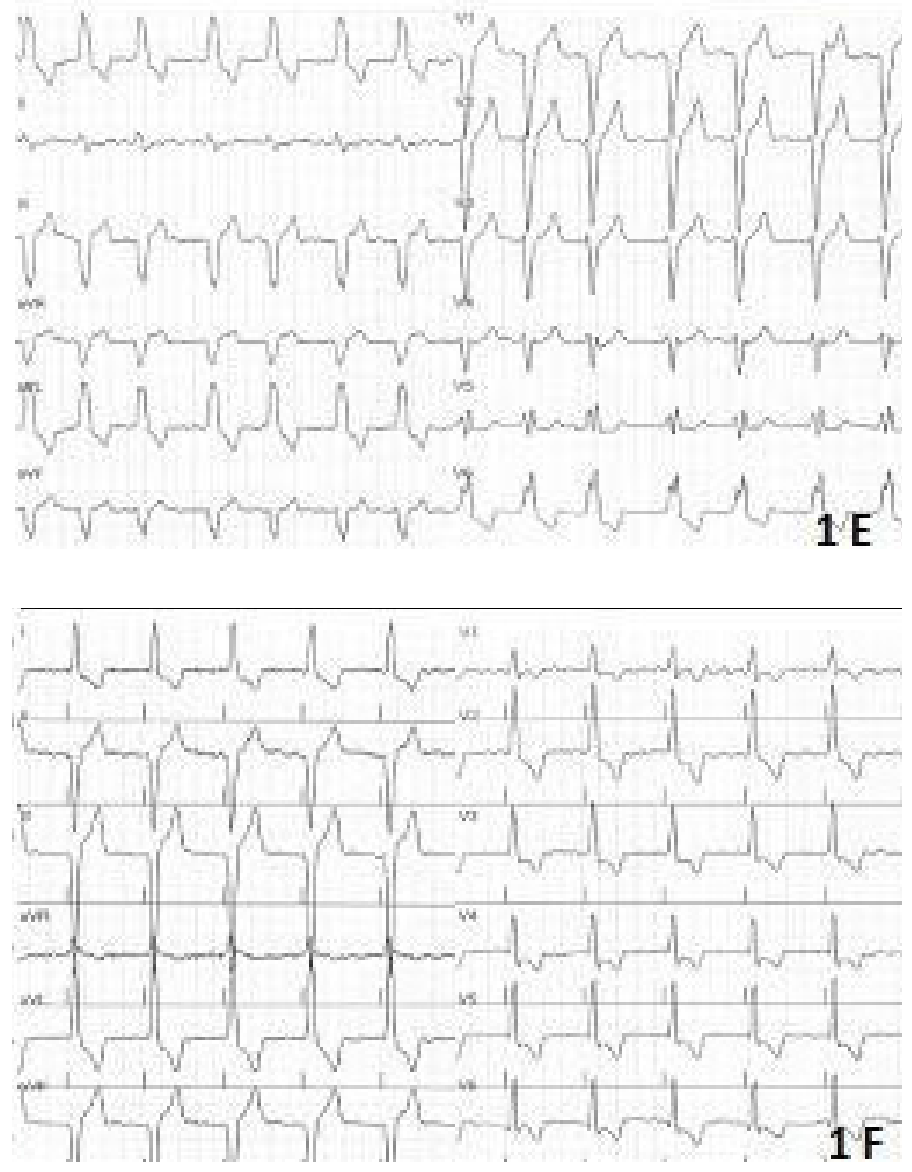

Figure 1E, F. Electrocardiogram: E. Before implantation - typical LBBB (QRS width $160 \mathrm{~ms}$ ). F. After implantation - biventricular pacing with narrowing of QRS complexes' width to $140 \mathrm{~ms}$.

$\mathrm{mm}$, respectively). The evolution of HF in our patient could only be explained with acquired iatrogenic LBBB. Due to HF in NYHA class III-IV with recent exacerbations, non-ischemic aetiology, female gender and LBBB with QRS complex width $>150 \mathrm{~ms}$, our patient seemed to be an ideal candidate for CRT implantation. Unexpectedly, during the surgery, we encountered PLSVC, which has never been described before. If the implantation had failed, the patient's prognosis would have been serious - the only treatment would have been epicardial lead placement surgery. However, in this case the surgery would have been hazardous because of the high risk of complications due to severe heart failure and adhesions after previous surgery.

\section{References}

1. Biffi M, Boriani G, Frabetti L, Bronzetti G, Branzi A. Left superior vena cava persistence in patients undergoing pacemaker or cardioverter-defibrillator implantation: a 10-year experience. Chest. 2001;120: 139-44, PMID: 11451829.
2. Irwin RB, Greaves M, Schmitt M. Left superior vena cava: revisited. Eur Heart J Cardiovasc Imaging. 2012;13:2 84-91, doi: 10.1093/ehjci/jes017.

3. Ratliff HL, Yousufuddin M, Lieving WR, et al. Persistent left superior vena cava: case reports and clinical implications. Int J Cardiol 2006;113: 242 -246, doi: 10.1016/j.ijcard.2005.08.067. 